\title{
ULTRASONIC ATTENUATION DURING THE MARTENSITIC TRANSFORMATION IN Cu-Zn-AI SHAPE MEMORY ALLOYS
}

\author{
LI. MANOSA, J.L. MACQUERON* and J.C. BABOUX* \\ Dept. Estructura i constituents de la Matèria, Facultat de Fisica, Universitat de Barcelona, Diagonal \\ 645, Barcelona, Catalonia, Spain \\ ${ }^{*}$ Laboratoire de Traitement du Signal et Ultrasons, INSA, Bât 502, 20 avenue Albert Einstein, \\ Villeurbanne, France
}

\begin{abstract}
We report measurements of the attenuation of longitudinal elastic waves, carried out using a broad-band immersion pulse-echo technique. The ultrasonic attenuation has been measured as a funcion of temperature and frequency during the martensitic transformation of monocrystalline and polycrystalline $\mathrm{Cu}-\mathrm{Zn}-\mathrm{Al}$ alloys. It has been shown that the most likely mechanism producing ultrasonic attenuation is the scattering of the ultrasonic waves by regions with different acoustic impedances.
\end{abstract}

\section{§ 1. INTRODUCTION}

The martensitic transformation undergone by copper-based shape-memory alloys has been investigated using a lot of different experimental techniques as, for example, electrical resistivity, differential scanning calorimetry, electron microscopy, etc... Ultrasonic techniques have also been used, mostly to examine the elastic behaviour of the high temperature phase ( $\beta$-phase) [1]. Nevertheless, no reliable measurements have been performed during the martensitic transformation itself, due to the fact that the growth of the martensitic phase results in a surface relief affecting the acoustic coupling between the sample and the piezoelectric transducer used to generate and detect the ultrasounds.

In a previous work [2] we analysed which was the effect of the surface relief in the measurement of the ultrasonic attenuation of longitudinal ultrasonic waves in $\mathrm{Cu}-\mathrm{Zn}-\mathrm{Al}$ polycrystals and single crystals undergoing martensitic transformations. The results showed that the temperature dependence of the ultrasonic attenuation could exhibit very different behaviour depending on whether the surface of the sample was flat in the $\beta$ or in the martensitic phase.

In this work we measured the ultrasonic attenuation of longitudinal waves using an experimental set-up for which the effects inherent to the surface relief were drastically reduced. It enabled to obtain the ultrasonic attenuation as a function of temperature and frequency during the martensitic transformation of $\mathrm{Cu}-\mathrm{Zn}-\mathrm{Al}$ single crystals and polycrystals. 


\section{§2. EXPERIMENTAL DETAILS}

Polycrystalline (Cu-24.3 $\mathrm{Zn}-9.0 \mathrm{Al}$ at \%) and monocrystalline (Cu-13.7 $\mathrm{Zn}-17.0 \mathrm{Al}$ at\%) samples were investigated. Their transition temperatures, $M_{s}$, were about $295 \mathrm{~K}$ and $300 \mathrm{~K}$ respectively. Samples were cylindrical with $10 \mathrm{~mm}$. thickness and $13 \mathrm{~mm}$. diameter for single crystals and $18 \mathrm{~mm}$. for polycrystals. After betatisation, polycrystals were kept for variable times at $1120 \mathrm{~K}$ in order to obtain samples having different grain sizes between them, ranging from 0.2 to $2 \mathrm{~mm}$. approximately. The single crystals were cut with their faces parallel to the (110) planes. The final preparation of the samples was achieved by mechanical polishing ( $1 \mu \mathrm{m}$ alumina powder).

Ultrasonic attenuation measurements were performed by a broad-band, immersion, pulse-echo method. The transition was thermally induced by heating and cooling the water bath where the sample and two identical broad-band transducers were immersed. The temperature was measured by a thermocouple directly attached onto the lateral surface of the sample. Details of the experimental system can be found elsewhere [3].

\section{§3. RESULTS}

The use of an immersion technique enabled a reduction of the effects associated with surface distortion in measuring ultrasonic attenuation. These effects were shown to be less than $0.5 \mathrm{~dB}$ over the frequency range studied [3].

Our investigations were restricted to the first transmitted ultrasonic pulse, from which, following the data treatment described in a previous work [3], the ultrasonic attenuation coefficient as a function of frequency and temperature can be obtained.

Figure 1 shows the ultrasonic attenuation coefficient as a function of temperature and frequency for a single crystal and two polycrystalline samples with average grain diameter of approximately 0.2 and $1 \mathrm{~mm}$.

For polycrystals the ultrasonic attenuation is higher in the $\beta$-phase than in the martensitic one, whereas for single-crystals the attenuation is higher in martensite than in the $\beta$-phase in which it can be considered negligible.

As revealed by figure 1, different behaviour for the ultrasonic attenuation is observed depending on the microstructure of the sample under investigation. For polycrystals with small grain size, the ultrasonic attenuation changes continuously through the martensitic tansformation (fig. 1a). As the grain size is increased, the ultrasonic attenuation coefficient vs. temperature curve shows a frequency dependent maximum at a temperature within the range of transformation (fig. 1b). This maximum is larger for larger grain sizes and becomes very remarkable during the transformation of single-crystals (fig. 1c). The magnitude of this maximum is independent of the amplitude of the ultrasound as well as of the heating/cooling rate. 

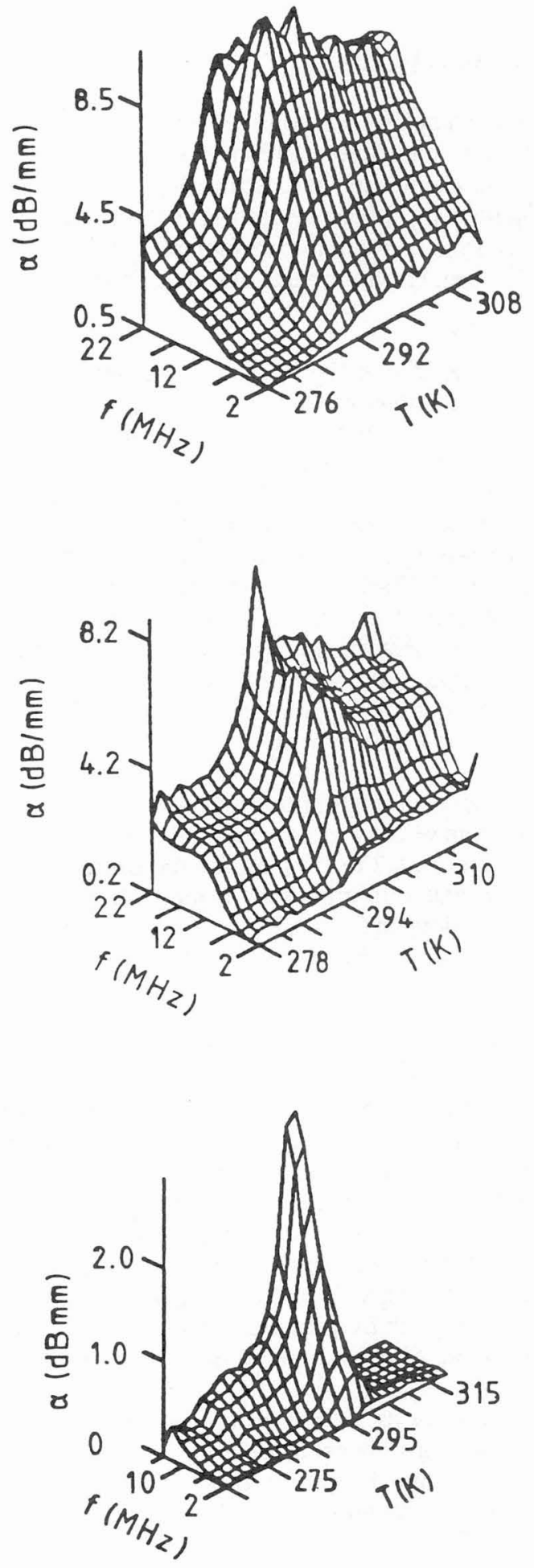

Fig. 1.a.- Ultrasonic attenuation as a function of temperature and frequency for a polycrystal with average grain size around $0.2 \mathrm{~mm}$.

Fig, 1.b.- Ulirasonic attenuation as a function of temperature and frequency for a polycrystal with average grain size around $1 \mathrm{~mm}$.

Fig. 1.c.- Ultrasonic attenuation as a function of temperature and frequency for a single crystal. 


\section{\$4. DISCUSSION}

To account for the experimental findings, we will consider the scattering of ultrasonic waves by regions with different acoustic impedances within a solid. The behaviour of ultrasonic attenuation coefficient associated to the scattering caused by elastic inhomogeneities is schematised in figure 2 [4]. It can be split into three different regions (labelled I, II and III), depending on the ratio between the wavelength of the ultrasound and the average diameter of the scattering element; maximum attenuation occurs when both magnitudes are similar.

Let us first consider the case of single crystals (fig 1c). In the high temperature phase, the crystal is elastically homogeneous and ultrasonic attenuation is caused only by absorption mechanisms, which indeed result in a very weak attenuation. As the transformation proceeds, a number of martensite and $\beta$ regions co-exist in the solid, producing reflections and refractions of the ultrasound at the interfaces and, therefore, a sharp increase in attenuation is observed on starting the transformation. On further cooling, $\boldsymbol{\beta}$ domains progressively transform into martensite ones, and, on average, the martensite domains are smaller. This implies that scattering passes towards region I (Rayleigh domain). In addition, for $\mathrm{Cu}-\mathrm{Zn}-\mathrm{Al}$, the martensitic phase is less anisotropic than the $\beta$ one [5]. Both factors imply that the ultrasonic attenuation must decrease as the transformation proceeds. Owing to the fact that ultrasonic attenuation is expected to be maximum when the wavelength is similar to the average size of the scattering elements, the peak for high frequencies must be located at low temperatures. This is in agreement with our measurements [3].

For polycrystals, ultrasonic attenuation is high in the $\beta$ phase due to the scattering of ultrasound by grains. Considering a mean velocity for longitudinal waves of $5000 \mathrm{~ms}^{-1}$, the wavelength of ultrasound ranges from approx. 0.2 to $2.5 \mathrm{~mm}$. for the frequency domain of our investigations. In the case of polycrystals with grain size around $0.2 \mathrm{~mm}$. (fig 1a), the scattering corresponds to the intermediate domain (region II), and increases with frequency, in agreement with phenomenological theories [4]. When the transformation takes place, the average size of the scattering elements becomes smaller and the scattering passes towards region I for all frequencies. As a result of this, the ultrasonic attenuation decreases continuously and is lower in the martensitic phase than in the $\boldsymbol{\beta}$ one.

When the grain size is around $1 \mathrm{~mm}$. (fig. 1b) the scattering corresponds to region II for low frequencies and to region III for high frequencies. As mentioned before, the ultrasonic attenuation increases with frequency in region II, but in region III it is expected to be independent of frequency. This behaviour is clearly observed in present experiments where a plateau is observed for high frequencies. As in the previous case, the effect of the martensitic transformation is to reduce the average size of the scattering elements. This signifies that, for low frequencies, the scattering will go from region II towards region I without showing any maximum; however, for high frequencies, the scattering will go through region II and a maximum must be detected, as indeed, found in present experiments.

Other mechanisms causing energy losses as, for example, damped vibration of interfaces or relaxational mechanisms have also been considered in order to explain the experimental observations. Considering a damped vibration of interfaces, the maximum in the attenuation should be comparable to that found in internal friction measurements; it 


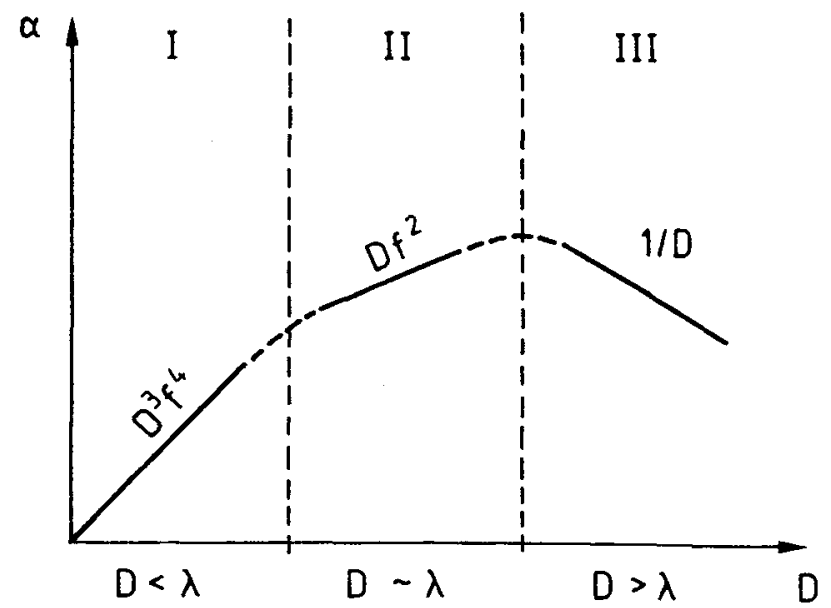

Fig. 2.- Ultrasonic attenuation coefficient corresponding to a scattering mechanism as a function of the average size of the scattering elements.

should be enhanced at low frequencies and dependent on both the amplitude of the strain and on the cooling rate [6]. The ultrasonic attenuation maximum found in this work is independent of the amplitude of the ultrasound and of the cooling rate; moreover, it decreases as frequency is reduced. These findings indicate that the mechanism causing maxima in ultrasonic attenuation are different from those causing internal friction maxima. On the other hand, if relaxational mechanisms are considered, the ultrasonic attenuation maximum is expected to shift towards higher temperatures when the frequency of ultrasound increases [7]. This prediction does not fit present observations.

To conclude, measurements of the attenuation of longitudinal waves during the martensitic transformation of monocrystalline and polycrystalline $\mathrm{Cu}-\mathrm{Zn}-\mathrm{Al}$ alloys, have shown that the most likely mechanism producing ultrasonic attenuation is the scattering of ultrasonic waves caused by regions with different acoustic impedances within the material.

\section{ACKNOWLEDGEMENTS}

Ll. Mañosa acknowledges partial financial support from CICyT, Spain, project MAT89-0748. Enlightening discussions with professors G.A. Saunders and P.J. Ford are also gratefully acknowledged.

\section{REFERENCES}

1.- $\quad$ B. Verlinden, L. Delaey, Acta Met 36 (1988) 1771.

2.- $\quad$ Ll. Mañosa, J.L. Macqueron, J.C. Baboux, Phil. Mag. A 59 (1989) 1277.

3.- $\quad$ Ll. Mañosa, J.L. Macqueron, J.C. Baboux, J. Phys: Condens. Matter 3 (1991) --.

4.- P. Papadakis, Physical Acoustics, Vol. IV-B, Ed. by W.P. Mason, Academic Press, 1968.

5.- $\quad$ G. Guénin, R. Pynn, D. Ríos-Jara, L Delaey, P.F. Gobin, phys. stat. sol. (a) 59 (1980) 553.

6.- G. Guénin, M. Morin, P.F. Gobin, Proc. ICIFUAS, Pergamon Press, Manchester (UK), 1979, p. 275.

7.- D.P. Almond, Q. Wang, J. Freestone, E.F. Lambson, B. Chapman, G.A. Saunders, J. Phys: Condens. Matter 1 (1989) 6853. 